\title{
Nonlinear relation between concentration and fluorescence emission of protoporphyrin IX in calibrated phantoms
}

\author{
Laure Alston \\ David Rousseau \\ Mathieu Hebert \\ Laurent Mahieu-Williame \\ Bruno Montcel
}




\title{
Nonlinear relation between concentration and fluorescence emission of protoporphyrin IX in calibrated phantoms
}

\author{
Laure Alston, ${ }^{a}$ David Rousseau, ${ }^{a}$ Mathieu Hebert, ${ }^{\mathrm{b}}$ Laurent Mahieu-Williame, ${ }^{\mathrm{a}}$ and Bruno Montcel ${ }^{\mathrm{a}, *}$ \\ aUniversity of Lyon, Universite Claude Bernard Lyon 1, UJM-Saint Etienne, CNRS, INSALyon, Inserm U1206, CREATIS UMR 5220, Lyon, France \\ 'University of Lyon, UJM-Saint Etienne, CNRS, Institut dOptique Graduate School, Laboratoire Hubert Curien, CNRS UMR5516, Saint-Étienne, \\ France
}

\begin{abstract}
ALA-induced protoporphyrin IX (PpIX) has shown its relevance in medical assisting techniques, notably in the detection of glioma (brain tumors). Validation of instruments on phantoms is mandatory and a standardization procedure has recently been proposed. This procedure yields phantoms recipes to realize a linear relationship between PpIX concentration and fluorescence emission intensity. The present study puts forward phantoms where this linear relationship cannot be used. We propose a model that considers two states of PpIX, corresponding to two different aggregates of PpIX, with fluorescence spectra peaking at 634 and $620 \mathrm{~nm}$, respectively. We characterize the influence of these two states on PpIX fluorescence emission spectra in phantoms with steady concentration of PpIX and various microenvironment parameters (surfactant, Intralipid or bovine blood concentration, and $\mathrm{pH}$ ). We show that, with fixed PpIX concentration, a modification of the microenvironment induces a variation of the emitted spectrum, notably a shift in its central wavelength. We show that this modification reveals a variation of proportions of the two states. This establishes phantom microenvironment regimes where the usual single state model is biased while a linear combination of the two spectra enables accurate recovering of any measured spectra. @ 2018 Society of Photo-Optical Instrumentation Engineers (SPIE) [DOI: 10.1117/1.JBO.23.9.097002]
\end{abstract}

Keywords: fluorescence spectroscopy; protoporphyrin IX; 5-aminolevulonic acid; phantom characterization.

Paper 170791RRR received Dec. 12, 2017; accepted for publication Sep. 5, 2018; published online Sep. 24, 2018.

\section{Introduction}

Fluorescence measurements are of considerable interest in medical-assisting techniques. ${ }^{1-3}$ Among them, 5-aminolevulonic acid (5-ALA)-induced protorporphyrin IX (PpIX) fluorescence offers the possibility to visualize tumor cells during neurosurgery. ${ }^{4-6}$ PpIX takes part in the biosynthesis of heme and thus naturally occurs in human bodies. Ingestion of 5-ALA, precursor in heme's biosynthesis, leads to temporary enhancement of PpIX concentration in glioma cells (glioma being tumors). ${ }^{7}$ PpIX emits a reddish fluorescence when excited under UV/blue light (around $405 \mathrm{~nm}$ ). This fluorescence has been used over the last 20 years as an intraoperative tool to assist neurosurgeons during glioma resection through the use of fluorescence microscopes. ${ }^{8}$ Various optical measurements of PpIX fluorescence have been performed. Those studies could be divided in two main approaches, either assessing qualitative or semiquantitative biomarkers related to PpIX fluorescence or quantitative concentration of PpIX. The first approach includes the basic imaging techniques that measure raw PpIX fluorescence intensity in wide field illumination. ${ }^{8}$ This technique is the actual clinical standard for PpIX-based surgical assistance. Some works proposed normalization procedures or other qualitative biomarkers to increase the robustness of PpIX fluorescence measurement. ${ }^{9-11}$ As for wide field clinical techniques, these methods illuminate and detect fluorescence exiting the same tissue surface area. Then, they mainly detect fluorescence emitted very close to the surface of the tissue.
The second approach aims at quantifying PpIX concentration. This relies on the assumption that the emitted PpIX fluorescence intensity is proportional to the concentration of PpIX. It uses fluorescence and reflectance spectroscopic measurements associated with light propagation models to correct the distorting effect of tissue and get quantitative information. ${ }^{12-14}$ Both approaches are relevant because all these measurements are intermediary steps toward the final aim of PpIX fluorescence measurements, which is the clustering of measurements into relevant pathological groups.

Relevant phantoms are needed for instrument validation and performance assessment of these methods. Recent works proposed a standardization of PpIX optical phantoms. ${ }^{15}$ They proposed a standardized PpIX phantom recipe to respect the proportionality between fluorescence intensity and PpIX concentration of the phantom. However, this phantom recipe does not consider a variation of the peak position in the fluorescence spectrum, whereas other studies have introduced it. ${ }^{10,11}$ Those studies either consider a wavelength shift of the peak intensity position ${ }^{10}$ or the presence of two states of PpIX with different fluorescence spectra, peaking at $634 \mathrm{~nm}$ and also at $620 \mathrm{~nm}$, respectively. ${ }^{11}$

For the qualitative methods, the correction of the distorting effect by tissue is not a critical issue. First, because they mainly detect fluorescence emitted very close to the surface of the tissue. Furthermore, they rely on qualitative biomarkers that are more robust to such distortion than the quantification of 
PpIX concentration. That is the reason why they do not consider an optical propagation model.

The present study aims at proposing PpIX phantoms recipes showing both states of PpIX peaking at $634 \mathrm{~nm}$ (State634) and also at $620 \mathrm{~nm}$ (State620). The study shows the switching from one state (of the fluorescence emission) to the other by changing the components and their concentration in the phantom. In this phantom study, the two states of PpIX are obtained by the two different aggregates of PpIX in aqueous solution. ${ }^{16}$ These phantoms are not intended to correspond to the optical properties of tissues, they aim at showing how biochemical conditions can modify the PpIX fluorescence spectrum. This mixing of two states induces a nonlinear relation between fluorescence intensity and PpIX concentration of the phantom. The presented data propose PpIX phantom recipe for fluorescence measurements based on two states of PpIX ${ }^{11}$ and on wavelength shift of the peak intensity position. ${ }^{10}$

\section{Materials and Methods}

\subsection{Optical Phantom Preparation}

Optical phantoms were aqueous solutions containing PpIX, dimethyl sulfoxide (DMSO), and phosphate buffered saline (PBS). PpIX concentration was set around $10^{-5} \mathrm{~mol} / \mathrm{L}$ to be close to in vivo concentrations. ${ }^{7,13}$ Additional components were sequentially added as proposed by Marois et al. ${ }^{15}$ to get various microenvironments of PpIX. Solutions were prepared by dissolving $6.1 \mathrm{mg}$ of PpIX (Sigma Aldrich, Saint Louis) into $10 \mathrm{~mL}$ of DMSO to get a stock solution at $1.2 \times 10^{-3} \mathrm{~mol} / \mathrm{L}$. Then, $1 \mathrm{~mL}$ of this stock solution was diluted into $80 \mathrm{~mL}$ of PBS to reach a concentration of PpIX of $1.5 \times 10^{-5} \mathrm{~mol} / \mathrm{L}$. The $\mathrm{pH}$ of this solution was then shift to 9 by addition of sodium hydroxide $(\mathrm{NaOH})$, giving a solution $S_{0}$. Minute addition of bovine blood (obtained from butcher), Tween 20 (Sigma Aldrich), or Intralipid 20\% (Fresenius Kabi) was independently performed starting from $S_{0}$. Volume variations due to addition of any items were $<0.4 \mathrm{~mL}$ and thus neglected. $\mathrm{pH}$ variations due to addition of Intralipid, Tween 20, or blood were also neglected since we stayed in basic media, with a pH between 8 and 9 . The $\mathrm{pH}$ of a fourth solution containing Intralipid, with a lipid volume fraction (LVF) of $6 \mathrm{ppm}$ was changed in the $\mathrm{pH}$ range of 5 to 9 by minute addition of hydrochloric acid $(\mathrm{HCl})$ or $\mathrm{NaOH}$ to study the influence of $\mathrm{pH}$ variations on PpIX emitted spectrum as proposed by Hope and Higham. ${ }^{17}$ Concentration of each studied item was regularly increased and emitted fluorescence was measured each time. The maximum concentration was chosen to reach the asymptote of State620 contribution, i.e., when the overall shape of the fluorescence emission spectrum stabilizes with increasing concentration.

\subsection{Spectroscopic Equipment}

Excitation for spectroscopic measurements was performed with a light-emitting diode (LED) (M405F1, Thorlabs) at $405 \mathrm{~nm}$, which corresponds to the maximum of the excitation spectrum of PpIX. ${ }^{16,18}$ Emitted light from LED (bandwidth at full width at half maximum $=16 \mathrm{~nm}$ ) was filtered by a low pass filter (cutoff wavelength $450 \mathrm{~nm}$ ) and injected into a $550-\mu \mathrm{m}$ core emitting optical fiber. The power output at the end of the fiber was $30 \mu \mathrm{W}$, yielding an irradiance of $26 \mathrm{~W} . \mathrm{m}^{-2}$ (considering that the emission surface is the fiber core section). Fluorescence emission was collected through a $550-\mu \mathrm{m}$ core collecting optical fiber. Output light was filtered by high pass filter (cutoff wavelength $485 \mathrm{~nm}$ ) and transmitted to a spectrometer (Maya2000pro Oceanoptics with a spectral resolution of $4 \mathrm{~nm}$ ). Emitting and collecting optical fibers were immersed into the phantom solution and slow agitation was kept in order to minimize photobleaching and optimize homogeneity of the solution. The fibers are pointing in two different directions that form an angle of $30 \mathrm{deg}$ between them. The fiber edges are positioned at $2 \mathrm{~mm}$ from the intersection of these two directions, leading to an optical path of $4 \mathrm{~mm}$.

\subsection{Data Processing}

Our processing steps were: (i) measurements consisted in a series of 20-s long measurements, each one with a 500-ms fluorescence measurement followed by a 500-ms background measurement (excitation light off). (ii) The emitted spectrum was obtained by subtracting the background from each measured spectrum to eliminate external stray light and by a summation of the 20 series. (iii) The emitted spectrum was low pass filtered by a zero phase squared cardinal sine window whose width correspond to the spectrometer's real optical spectral resolution. (iv) The filtered emitted spectrum $S(\lambda)$ was fitted (lsqcurvefit; Matlab, MathWorks Inc.) according to Eq. (1) in the 608- to 637-nm spectral range

$S(\lambda)=\alpha_{620} S_{620}(\lambda)+\alpha_{634} S_{634}(\lambda)$,

where $S_{634}(\lambda)$ and $S_{634}(\lambda)$ are the normalized fluorescence emission spectra of State634 and State620 obtained in phantom, respectively, considered as the reference spectra and shown Fig. 1. $\alpha_{634}$ and $\alpha_{620}$ are the contributions of State634 and State620, respectively, to the total emitted fluorescence and takes into account the molar quantum yield of each state, their concentrations, and other known contributions to fluorescence intensity (the incident light intensity, the optical pathlength, and the molar extinction coefficients). The 608 to $637 \mathrm{~nm}$ range was chosen to simplify the previous model, ${ }^{11}$ as the photoproducts spectra are difficult to evaluate and can highly distort the spectrum in the 637 to $750 \mathrm{~nm}$ range. Normalized fluorescence emission spectra, $S_{634}(\lambda)$ and $S_{620}(\lambda)$, were obtained in phantoms. The measured spectra were normalized by their total fluorescence intensities in the 608 to $637 \mathrm{~nm}$ range, see Fig. 1 where units are arbitrary. $S_{620}(\lambda)$ was obtained into the solution $S_{0}$ as proposed in Ref. $16 S_{634}(\lambda)$ was obtained

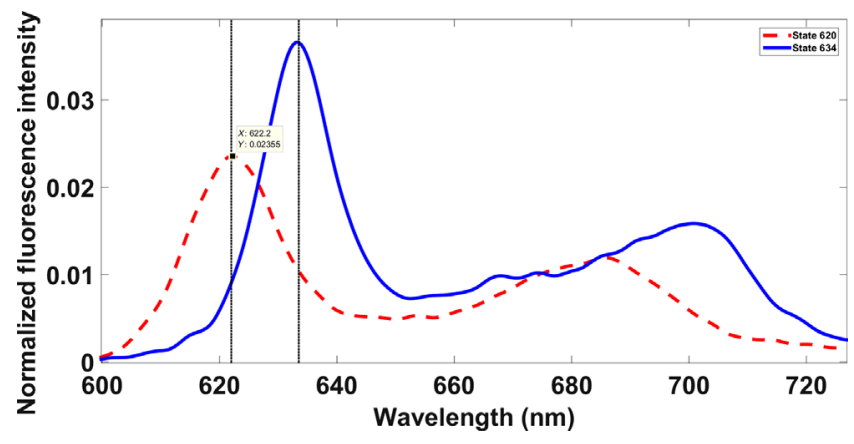

Fig. 1 Fluorescence emission spectra of the two states of PpIX considered as reference spectra, normalized by their total intensity between 608 and $637 \mathrm{~nm}$ and named $S_{620}(\lambda)$ (red dotted line) and $S_{634}(\lambda)$ (blue line) thereafter. Black markers localize their maxima (respectively $622 \mathrm{~nm}$ and $633.5 \mathrm{~nm}$ ). 
by addition of $\mathrm{HCl}$ into $S_{0}$ to reach a $\mathrm{pH}$ of 5 and then addition of $1 \mathrm{~mL}$ of Tween 20 and $1 \mathrm{~mL}$ of Intralipid, as proposed in Ref. 15.

This fit yields the two parameters $\alpha_{634}$ and $\alpha_{620}$. As in Ref. 11 those parameters are not quantified and given in arbitrary units. To investigate the biomarker proposed in Ref. 10, i.e., the wavelength shift of the peak position, we also studied the evolution of the spectrum's central wavelength. The central wavelength was defined as the mean wavelength of the interval representing more than $80 \%$ of the maximum's fluorescence intensity. For comparison purpose between the measured central wavelength and the fitting procedure, we also evaluated the computed central wavelength of fitted $S(\lambda)$. Since $\alpha_{i}$ does not only include the concentration of each state but also some unknown parameters contributing to the total fluorescence, we decided to show the relative contribution of each state to the total fluorescence.

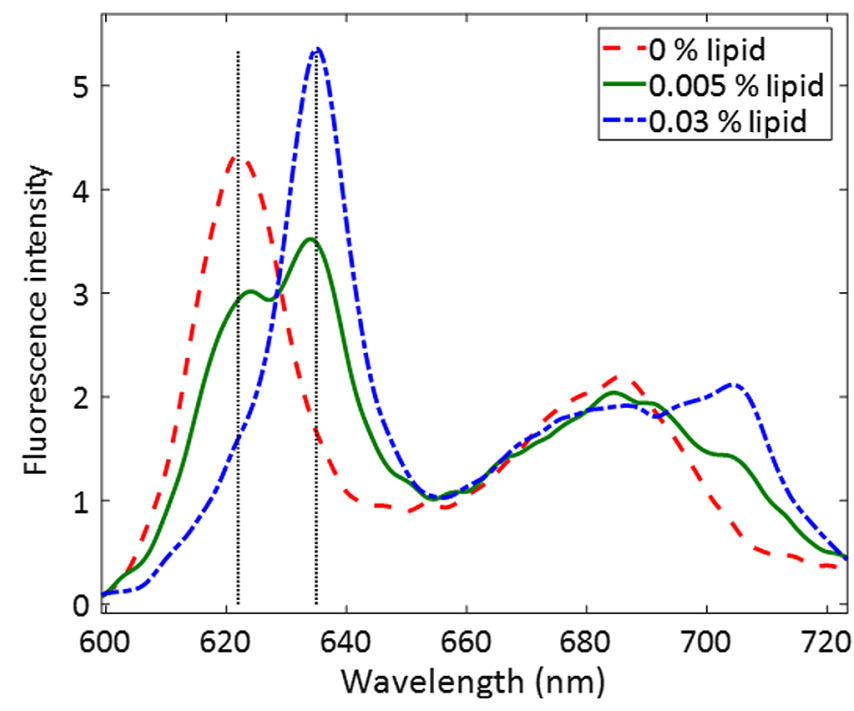

Fig. 2 Emission spectra of PpIX in aqueous solution with various LVF (\%). Black markers were added to localize maxima (622 and $635 \mathrm{~nm}$, respectively).

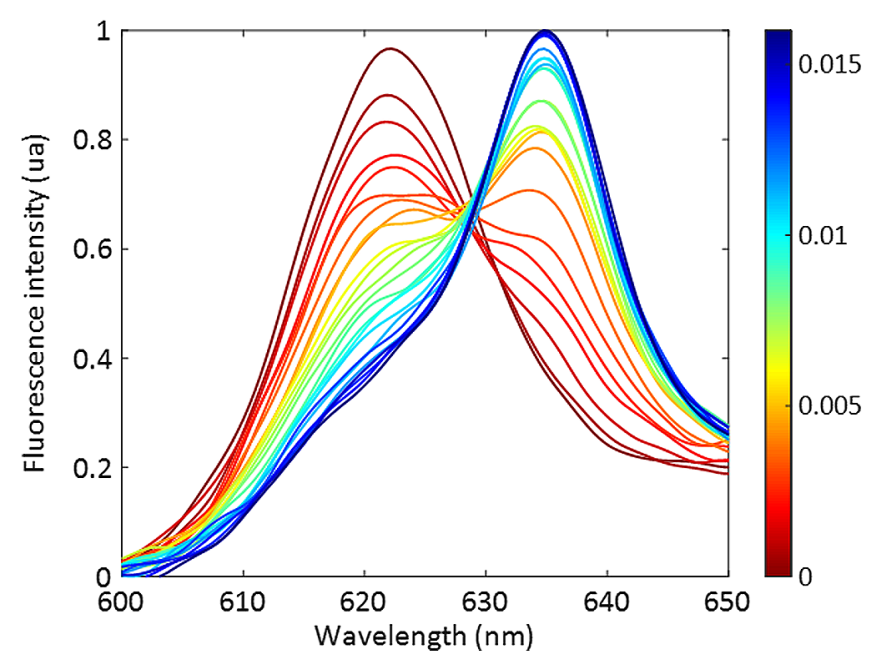

(a)
Thus, each computed spectrum was modeled as

$S(\lambda)=(1-\beta) S_{620}(\lambda)+\beta S_{634}(\lambda)$,

where

$\beta=\frac{\alpha_{634}}{\alpha_{620}+\alpha_{634}}$,

and $\beta$ varied from 0 to 1 . Thus, $\beta$ does not reveal the concentration of the State634 but its relative contribution to total fluorescence and reciprocally $(1-\beta)$ for the State620.

\section{Results}

Figure 2 shows three fluorescence emission spectra of PpIX dissolved into DSMO and diluted into PBS with three increasing LVF: $0 \%, 0.005 \%$, and $0.03 \%$.

This figure shows a shift from a fluorescence spectrum with a peak around $620 \mathrm{~nm}$ (no lipid) to a fluorescence spectrum with a peak at $634 \mathrm{~nm}(\mathrm{LVF}=0.03 \%)$ passing by an intermediary fluorescence spectrum showing two local maxima ( $\mathrm{LVF}=$ $0.005 \%$ ). More fluorescence spectra collected with increasing concentration of Intralipid are shown Fig. 3(a). Figure 3 shows intermediary spectra appearing with the increase of the concentration of Intralipid.

Addition of surfactant highlights the same shift [see Fig. 4(a)] and diminution of $\mathrm{pH}$ values also exhibits this shift with moreover a diminution in total amount of emitted light see [Fig. 6(a)]. Addition of blood enables shift from a spectrum with a main peak around $620 \mathrm{~nm}$ to intermediary emitted spectra but signal-to-noise ratio becomes too small to process data with more blood [see Fig. 5(a)].

The graphics on Figs. 3-6 show all emitted spectra of the different phantoms (a) and the contribution of the two states to total fluorescence, obtained by the fit described in Eq. (1) (b). For readability, contributions $\alpha_{634}$ and $\alpha_{620}$ have been normalized by the initial contribution of State620, obtained with solution $S_{0}$, on each graphic in (b) (i.e., the normalized contributions equal $\alpha_{634} / \alpha_{620 \text {,initial }}$ and $\left.\alpha_{620} / \alpha_{620 \text {,initial }}\right)$. Fluorescence spectra in (a) graphics are emitted by each phantom starting

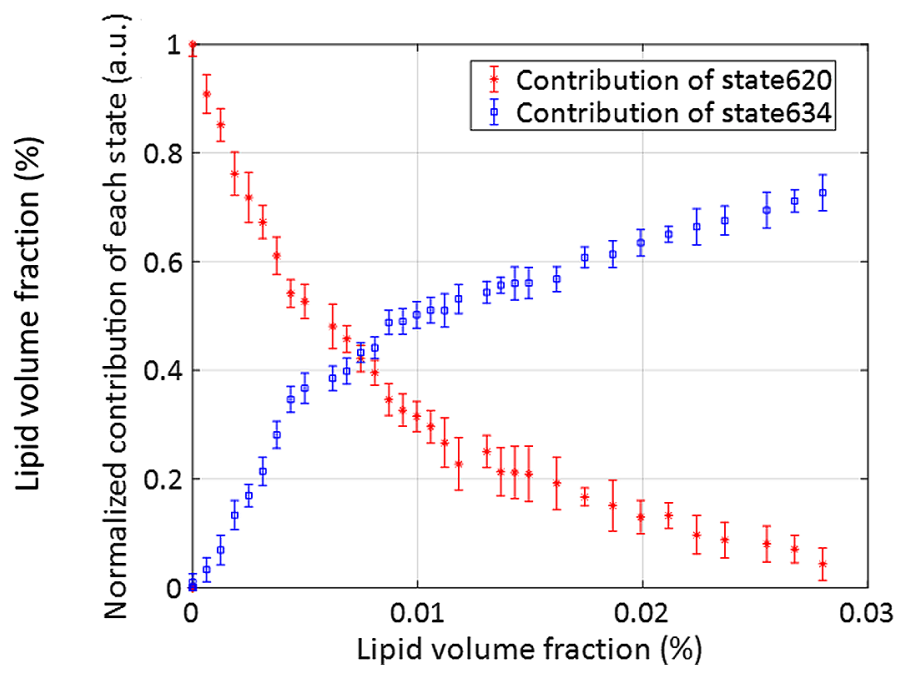

(b)

Fig. 3 (a) Emission spectra of PpIX in aqueous solution with increasing LVF (\%) and (b) normalized contribution of each state (arbitrary units) obtained by a linear fit of those emitted spectra [presented Eq. (1)]. 


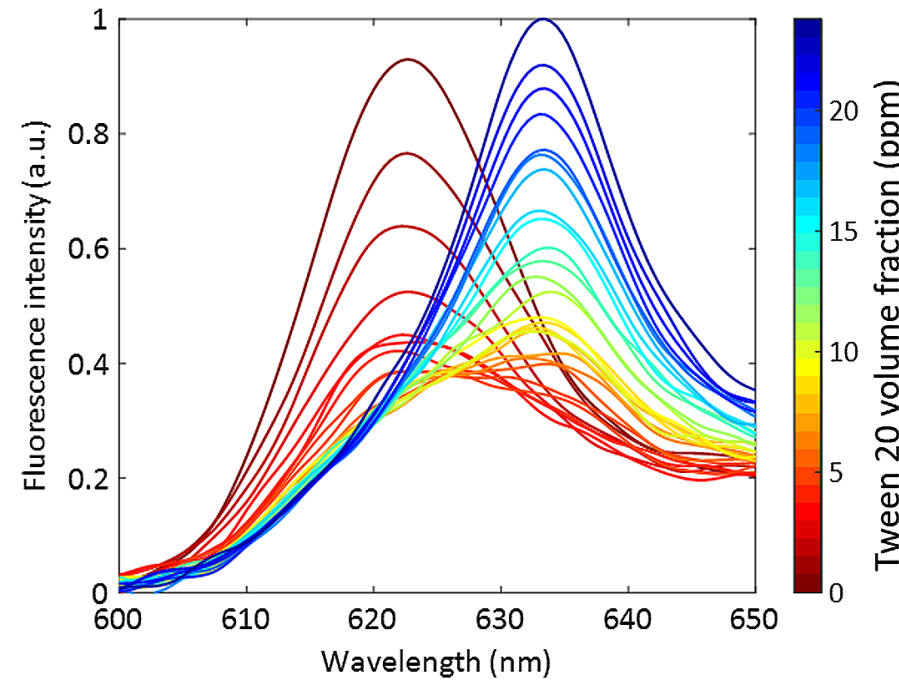

(a)

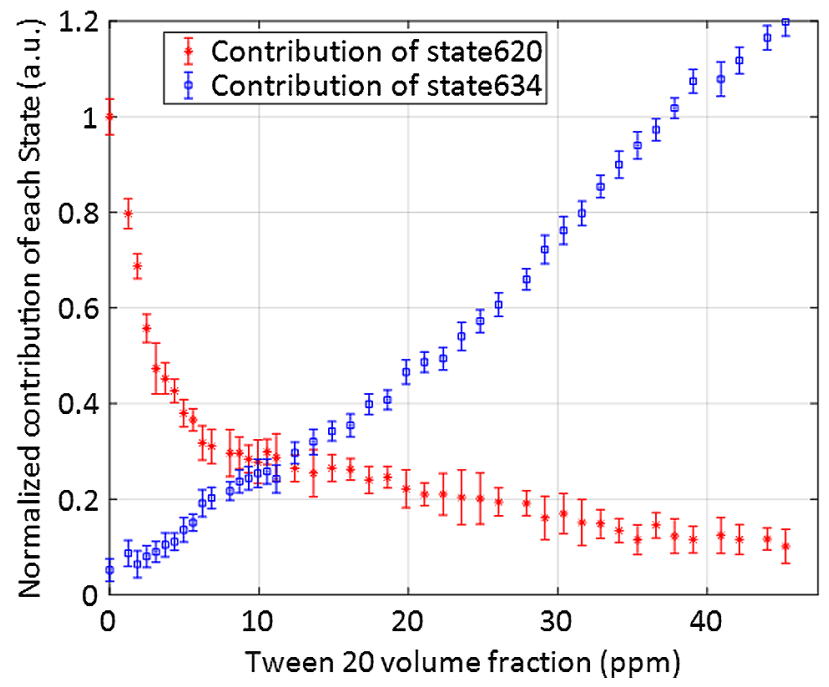

(b)

Fig. 4 (a) Emission spectra of $\mathrm{PpIX}$ in aqueous solution with increasing volume fraction (parts-permillion) of Tween 20 and (b) normalized contribution of each state (arbitrary units) obtained by a linear fit of those emitted spectra [presented Eq. (1)].

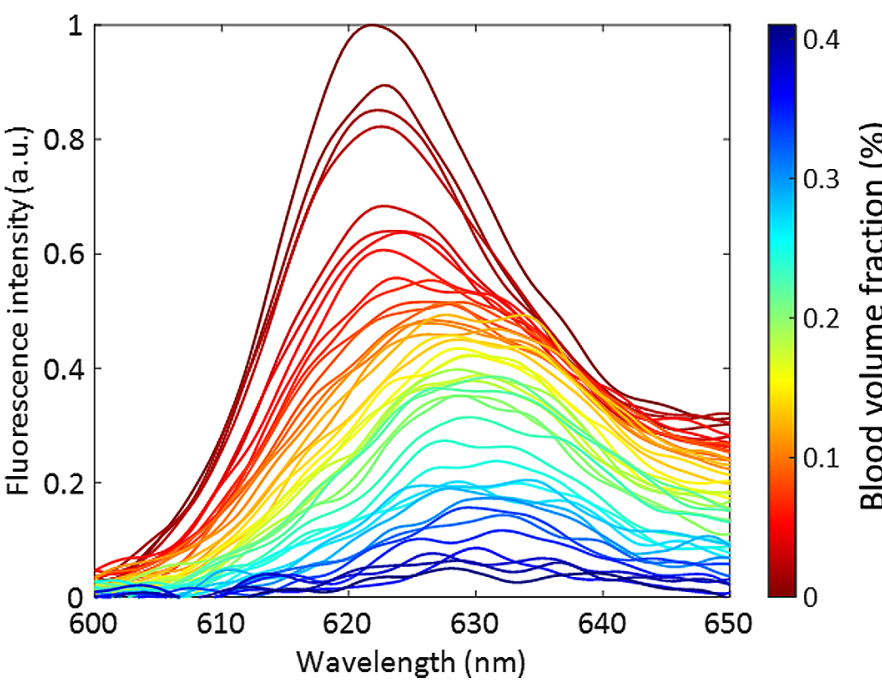

(a)

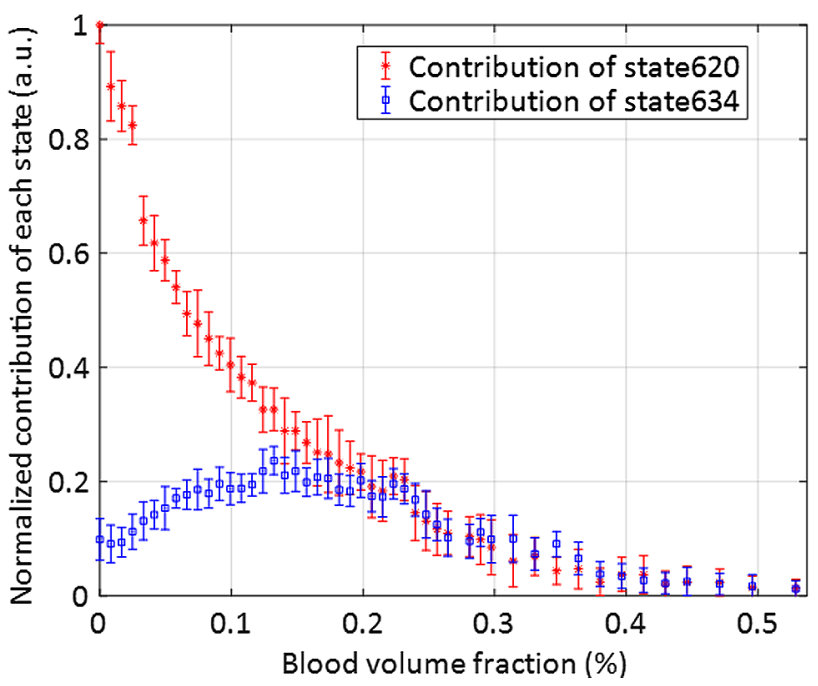

(b)

Fig. 5 (a) Emission spectra of $\mathrm{PpIX}$ in aqueous solution with increasing volume fraction (\%) of bovin blood and (b) normalized contribution of each state (arbitrary units) obtained by a linear fit of those emitted spectra [presented Eq. (1)].

from $S_{0}$ to a concentration of added item twice the concentration where both states have an equal contribution. For instance, spectra in 3(a) are plotted for LVF from $0 \%$ to $0.016 \%$ since the dominance changeover is around an LVF of $0.016 \%$. For Figs. 3-6, fluorescence spectra are normalized by the maximum value of all the plotted spectra.

Fluorescence emission of the four phantoms displays an analogous global trend. Charts in (a) display a shift of the maximum's wavelength from $620 \mathrm{~nm}$ to a value close to $634 \mathrm{~nm}$, passing by intermediary values, as already described. Charts in (b) display a shift from overwhelming contribution of State620 in total fluorescence to contribution of both states and finally to dominant contribution of State634 (if signalto-noise ratio enables it) with increasing concentration of
Intralipid, Tween 20, or blood (before a fall in signal-to-noise ratio) and decreasing $\mathrm{pH}$.

Focusing on (b) of Figs. 3-6, contribution of State620 drops from 1 to nearly 0 in all cases, whereas contribution of State634 varies in a different manner in the four studied solutions. Figures 3 and 4 show the apparition of State634 with a final contribution comparable to the initial contribution of State 620 in the presence of Intralipid (reaching $73 \%$ of $\alpha_{620 \text {,initial }}$ ) and even higher in presence of Tween 20 (reaching $120 \%$ of $\alpha_{620 \text {,initial }}$. Contribution of State634 in Fig. 5 intensifies until a blood volume fraction of $0.15 \%$. Beyond this limit, signalto-noise ratio becomes too low to distinguish the two contributions, which both fall down to zero. Finally, Fig. 6 highlights that State634 becomes predominant toward State620 in acid 


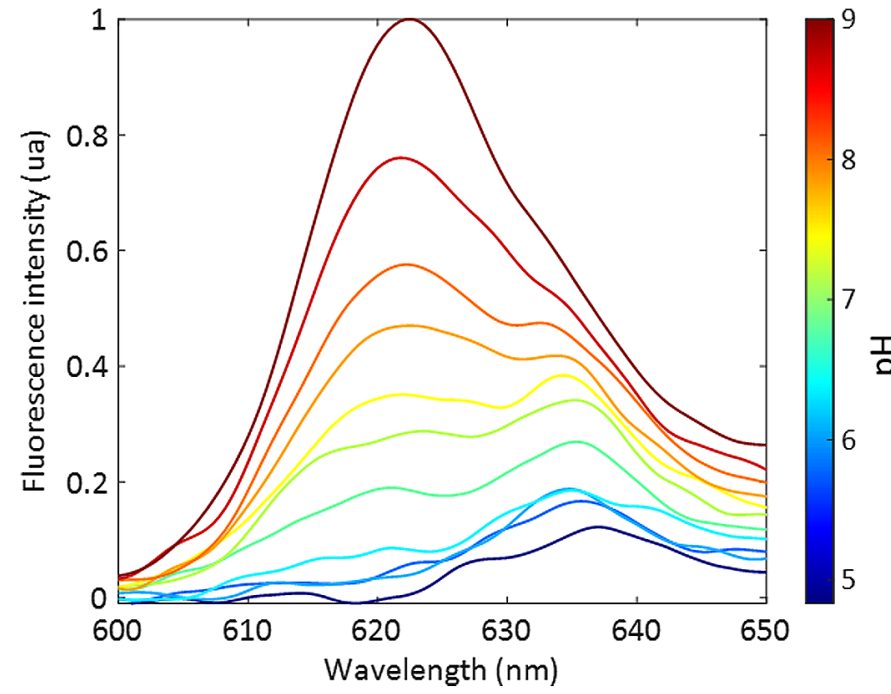

(a)

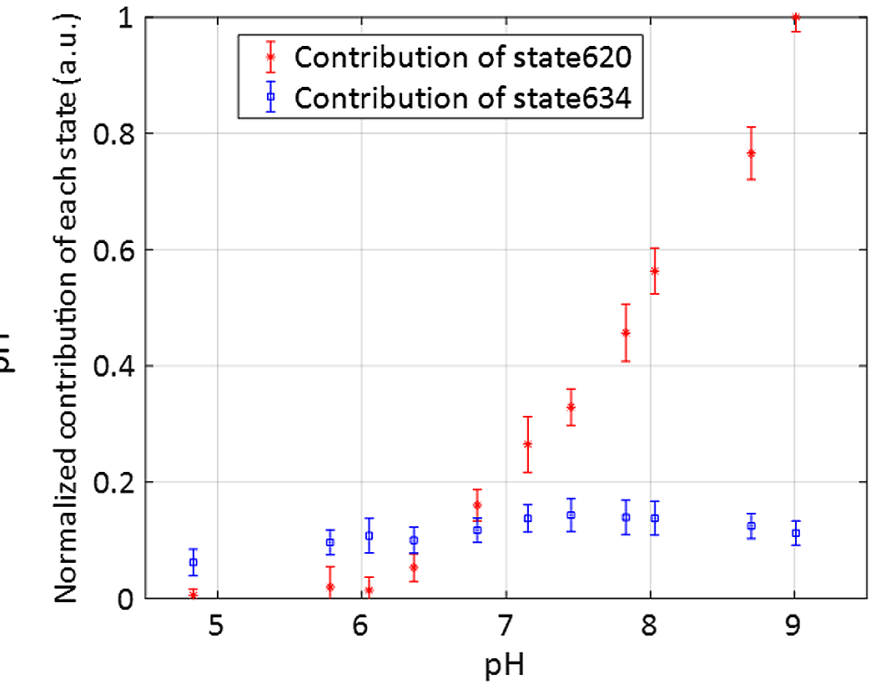

(b)

Fig. 6 (a) Emission spectra of PpIX in aqueous solution with increasing $\mathrm{pH}$ and (b) normalized contribution of each state (arbitrary units) obtained by a linear fit of those emitted spectra [presented Eq. (1)].

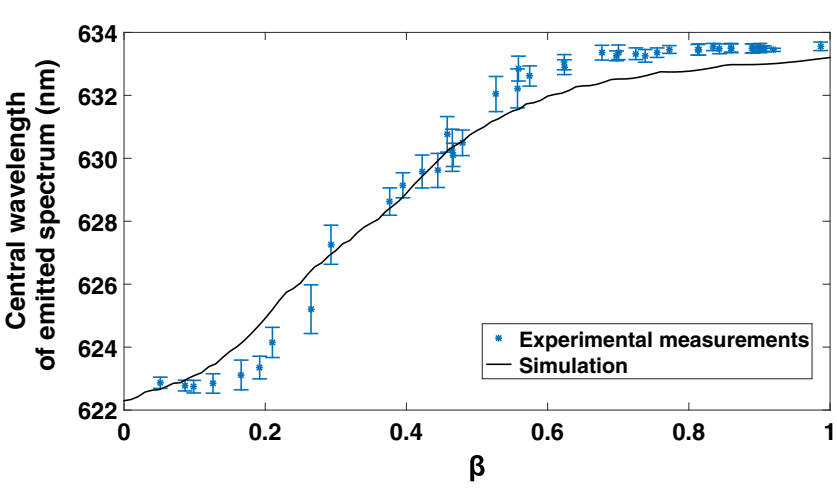

Fig. 7 Shift of fluorescence spectrum central wavelength with increasing $\beta$ in measured (stars with standard deviation) and simulated (dark line) spectra.

medium but its contribution stays below the initial contribution of State620.

Figure 7 shows this shift by displaying the changeover of the fluorescence emitted spectrum's central wavelength versus relative contribution of State634 (i.e., $\beta$ ) in a solution with increasing concentration of Tween 20 and also in computed spectra. Relative contribution of State634 $(\beta)$ was derived straightforward from Eq. (2) for computed spectra. For experimental measurements, $\beta$ was obtained with Eq. (3) after the fit yielding $\alpha_{620}$ and $\alpha_{634}$. This figure shows that the central wavelength shifts with increasing contribution of State634 and that the global shape of experimental contribution of State634 follows the pattern of simulated contribution of State634. However, some discrepancies appear near the asymptote when reaching State634. These discrepancies are due to the choice of the reference spectra for State634. It is complicated to decorrelate State634 and State620 spectra one from another. The discrepancies appear mainly on the State634 asymptote because it is very likely that our reference spectra are not totally independent from each other.

\section{Discussion}

This work enables us to propose phantom recipe in order to take into account the presence of a second state of PpIX already shown in vitro and mimicking fluorescence emission spectrum found in vivo in gliomas. ${ }^{11,16,17}$ Our results first confirm that PpIX into aqueous alkaline solution without any additional component shows a weak fluorescence spectrum with a main peak around $620 \mathrm{~nm}$, which corresponds to a particular aggregation of PpIX, as introduced by Melo and Reisaeter. ${ }^{16}$ The addition of some component enables us to get the strong and wellknown fluorescence spectrum with a main peak around $634 \mathrm{~nm}$, which corresponds to a different aggregate of PpIX, as already shown. ${ }^{15,16}$ What is more, those results show that, while concentration of PpIX remains constant, contribution of State634 varies and that the consideration of a linear link between fluorescence of State634 and concentration of PpIX is biased in those phantoms. Nonetheless, our results show that the use of a linear combination of the two states responds to the issue.

It should be emphasized that this bias can be prominent because fluorescence efficiency of State620 is considerably smaller than fluorescence efficiency of State634. ${ }^{18}$ The concentration of PpIX belongs to the range of concentrations studied by Marois et al.; ${ }^{15}$ nonetheless, they focused on phantoms where fluorescence of State634 outperforms fluorescence of State620. They also showed that fluorescence intensity degrades by $98 \%$ under some circumstances due to PpIX aggregation. This confirms the difference in efficiency between the two aggregates of PpIX.

Previous works have shown the presence of the second peak of fluorescence of PpIX at $620 \mathrm{~nm}$ in tissues ${ }^{11,14,19,20}$ or cell culture. ${ }^{17,21}$ But its origin is still an open issue. Some works support the assumption that the origin of the peak at $620 \mathrm{~nm}$ in cell culture is a different aggregate of PpIX. ${ }^{17}$ In addition, other works ${ }^{14,21}$ explained it by precursors of uroporphyrins or coproporphyrins. However, these studies either injected 5-ALA into bacteria or studied human brain samples that enabled the biosynthesis of heme in both cases. In this study, fluorescence at $620 \mathrm{~nm}$ appears from PpIX into aqueous solutions and could not be attributed to uroporphyrin or coproporphyrin. 
Other works ${ }^{10}$ also showed that the shift in the peak intensity of PpIX fluorescence could contain relevant information for the identification of tumor characteristics. We show here that a similar shift can also be reproduced in optical phantoms, see Fig. 7, and is attributed to different contributions of both states of PpIX.

Beyond the presence of the two states, Fig. 3 shows an asymptotic trend of the elevation of the contribution of State 634 for LVF above $0.03 \%$. This asymptote could denote the proportionality between fluorescence intensity and concentration of State 634 commonly used ${ }^{13}$ and underlined by Marois et al. with around 10 times higher LVF than that in our study. ${ }^{15}$ This proportionality would have been expected in our work if the increase in LVF had gone on but this was not the target of our work. This asymptote does not appear in graph of Fig. 4 with increasing amount of Tween 20, but there again the goal was not to reveal a proportional link between PpIX concentration and fluorescence of State634 but rather the necessity to consider weaker fluorescence of PpIX in phantoms. Marois et al. showed that this linearity appears with more than $0.1 \%$ Tween 20 volume fraction (TVF) and we added $4.5 \times 10^{-3} \%$ TVF, which is below their limit of $0.1 \%$ TVF. Nonetheless, there seems to be a linear relationship between increasing amount of surfactant and fluorescence of State634 in Fig. 4(b). This could be explained by hydrophobicity of PpIX and micelle formation or dissolution of aggregates of PpIX with the introduction of surfactant, as already discussed. ${ }^{7,11,15,16}$ Finally, the graphs of Fig. 5 show that there is no fluorescence beyond a blood volume fraction (BVF) of $0.3 \%$, whereas Marois et al. used until 3\% BVF. This discrepancy might be explained by the different experimental setup with a longer optical path length in our study, by the concentration of hematocrits in the blood used and by the need to add surfactant or Intralipid to prevent aggregate and thus to get fluorescence of State634 as done by Marois et al. In the end, the graphs in Fig. 6 also reveals a change in the physical state of PpIX with decreasing $\mathrm{pH}$ as proposed by Ref. 16. Nonetheless, fluorescence of State634 is weaker than fluorescence of State620 with only a variation of $\mathrm{pH}$. Addition of surfactant or Intralipid is necessary to amplify fluorescence intensity of State634, as recalled by Marois et al. ${ }^{15}$

The fitting model focused the range 608 to $637 \mathrm{~nm}$, where we assume that only PpIX contributes to emitted fluorescence. At higher wavelength than $637 \mathrm{~nm}$, variations in emitted spectra can be highly distorted by photoproducts or other chemical process. And the measurement of photoproducts spectra can be difficult. Moreover, the difference between $S_{620}(\lambda)$ and $S_{634}(\lambda)$ spectra is mainly focused in the range 608 to $637 \mathrm{~nm}$ where fluorescence intensity is the highest. Consequently, we found this fitting model well suited to differentiate the two states of PpIX.

\section{Conclusion}

Quantification of 5-ALA-induced PpIX is a new challenge in medical imaging, especially in neurosurgery to help the discrimination of tumor cells. A standardization procedure of optical phantom recipe has been proposed in previous works ${ }^{15}$ to respect the proportionality between fluorescence intensity and PpIX concentration of the phantoms. However, this standardization did not consider methods not based on this assumption. ${ }^{10,11}$ We showed in this work that fluorescence of the State620 contributes to PpIX emission fluorescence in different phantoms. It has been shown previously in gliomas ${ }^{11}$ that a modification of PpIX spectrum in this $620 \mathrm{~nm}$ area was linked to the pathological status of tissue. Thus, this $620 \mathrm{~nm}$ area should be considered carefully for PpIX fluorescence investigation in gliomas. We illustrated that the emitted spectrum of PpIX differs with microenvironment and that this variation denotes the presence of the two states of PpIX with varying relative contribution to total emitted fluorescence of PpIX. In addition, we showed that total fluorescence emission by PpIX is a linear combination of two reference spectra and thus, that the use of only one fixed emission spectrum as a spectral reference for PpIX is biased in some microenvironments. Consequently, this work proposes protoporphyrin IX phantoms recipe in order to take into account the presence of a second state of PpIX. This could be of real importance in the aim of getting PpIX spectral measurements methods into the standard tools in glioma resection clinical routine.

\section{Disclosures}

The authors have no relevant financial interests in this article and no potential conflicts of interest to disclose.

\section{Acknowledgments}

LABEX PRIMES (ANR-11-LABX-0063) of Université de Lyon, within the program "Investissements d'Avenir" (ANR11-IDEX-0007) operated by the French National Research Agency (ANR); Cancéropôle Lyon Auvergne Rhône Alpes (CLARA) within the program "OncoStarter"; France Life Imaging (FLI).

\section{References}

1. V. Ntziachristos, "Fluorescence molecular imaging," Annu. Rev. Biomed. Eng. 8, 1-33 (2006).

2. C. Chi et al., "Intraoperative imaging-guided cancer surgery: from current fluorescence molecular imaging methods to future multi-modality imaging technology," Theranostics 4(11), 1072-1084 (2014).

3. C. Ewelt et al., "Fluorescence in neurosurgery: its diagnostic and therapeutic use. Review of the literature," Photochem. Photobiol. 148, 302309 (2015).

4. X. Su et al., "Fluorescence-guided resection of high-grade gliomas: a systematic review and meta-analysis," Photodiagn. Photodyn. Ther. 11, 451-458 (2014).

5. H.-A. Leroy et al., "Fluorescence guided resection and glioblastoma in 2015: a review," Laser Surg. Med. 47, 441-451 (2015).

6. W. Stummer et al., "5-aminolevulinic acid-derived tumor fluorescence: the diagnostic accuracy of visible fluorescence qualities as corroborated by spectrometry and histology and postoperative imaging," Neurosurgery 74, 310-320 (2014).

7. A. Johansson et al., "5-aminolevulinic acid-induced protoporphyrin IX levels in tissue of human malignant brain tumors," Photochem. Photobiol. 86, 1373-1378 (2010).

8. W. Stummer et al., "Fluorescence-guided surgery with 5-aminolevulinic acid for resection of malignant glioma: a randomised controlled multicentre phase III trial," Lancet Oncol. 7(5), 392-401 (2006).

9. N. Haj-Hosseini et al., "Optical touch pointer for fluorescence guided glioblastoma resection using 5-aminolevulinic acid," Laser Surg. Med. 42, 9-14 (2010).

10. T. Ando et al., "Precise comparison of protoporphyrin IX fluorescence spectra with pathological results for brain tumor tissue identification," Brain Tumor Pathol. 28(1), 43-51 (2011).

11. B. Montcel et al., "Two-peaked 5-ALA-induced PpIX fluorescence emission spectrum distinguishes glioblastomas from low grade gliomas and infiltrative component of glioblastomas," Biomed. Opt. Express 4(4), 548-558 (2013).

12. A. Kim et al., "Quantification of in vivo fluorescence decoupled from the effects of tissue optical properties using fiber-optic spectroscopy measurements," J. Biomed. Opt. 15(6), 067006 (2010). 
13. P. A. Valdés et al., "Quantitative fluorescence in intracranial tumor: implications for ALA-induced PpIX as an intraoperative biomarker: clinical article," J. Neurosurg. 115, 11-17 (2011).

14. M. Zanello et al., "Multimodal optical analysis discriminates freshly extracted human sample of gliomas, metastases and meningiomas from their appropriate controls," Sci. Rep. 7, 41724 (2017).

15. M. Marois et al., "Characterization and standardization of tissue-simulating protoporphyrin IX optical phantoms," J. Biomed. Opt. 21, 035003 (2016).

16. T. B. Meløand and G. Reisaeter, "The physicochemical state of protoporphyrin IX in aqueous solution investigated by fluorescence and light scattering," Biophys. Chem. 25(1), 99-104 (1986).

17. C. K. Hope and S. M. Higham, "Evaluating the effect of local pH on fluorescence emissions from oral bacteria of the genus Prevotella," J. Biomed. Opt. 21(8), 084003 (2016).
18. G. I. Lozovaya, Z. Masinovsky, and A. A. Sivash, "Protoporphyrin IX as a possible ancient photosensitizer: spectral and photochemical studies," Origins Life Evol. Biospheres 20(3), 321-330 (1990).

19. W. Dietel et al., "5-aminolaevulinic-acid-induced formation of different porphyrins and their photomodifications," Lasers Med. Sci. 12(3), 226236 (1997).

20. G. Barron et al., "Porphyrin profile in four human cell lines after supplementation with 5-aminolaevulinic acid and its methyl ester," Photodiagn. Photodyn. Ther. 10(4), 654-663 (2013).

21. W. Dietel et al., "5-aminolaevulinic acid (ALA) induced formation of different fluorescent porphyrins: a study of the biosynthesis of porphyrins by bacteria of the human digestive tract," Photochem. Photobiol. 86, 77-86 (2007).

Biographies for the authors are not available. 